\title{
EPR (Electronic Patient Record) Laboratory - Simulated Environment to Learn about a Hospital EPR System
}

\author{
Kayo Watanabe
}

Department of Health Informatics

Kawasaki University of Medical Welfare

288 Matsushima, Kurashiki City, Okayama 701-0193, Japan

E-mail: k-watanabe@mw.kawasaki-m.ac.jp

\section{Mihoko Okada*}

Department of Health Informatics

Kawasaki University of Medical Welfare

288 Matsushima, Kurashiki City, Okayama 701-0193, Japan

E-mail: m-okada@mw.kawasaki-m.ac.jp

\section{Kazuko Yamamoto}

Office of Healthcare Information Technologist

Japan Association of Medical Informatics

8F Boa-Hongo, 3-42-5 Hongo, Bunkyo-ku, Tokyo 113-0033, Japan

E-mail: yam-sim@umin.net

*Corresponding author

\begin{abstract}
The "Electronic Patient Record (EPR) Laboratory" is a computer based self-learning system developed for students to acquire practical skills and knowledge required to deal with EPRs. The system is designed to supplement conventional lectures on health information systems given as part of our undergraduate curriculum. Using the Laboratory, the students may learn not only operations of EPR systems but also the subjects connected with patient information handling, including privacy, security and health information ethics. The EPR Laboratory is composed of an eLearing system and an EPR system. The learning materials are arranged in units in the eLearning system, and in each unit, the student learns the materials and the EPR operations through practice. Tests are given at each end of unit, and if a student failed a test, the system shows which questions were answered incorrectly and indicates which parts of the unit he/she should review. For this purpose, we introduced a structure to the learning materials based on an information model. In this paper, the overview of the system, the simulated environment to learn patient flow, information flow and hospital workflow, fundamental EPR operations, and structured learning materials for the test and review cycle are described.
\end{abstract}

Keywords: Self-Learning system; Electronic patient record; Simulated environment; Health information professional; Health information ethics

Biographical notes: Kayo Watanabe received B.S degree in pedagogy, M.S in engineering, and $\mathrm{Ph} . \mathrm{D}$ in medical informatics. She served as a teacher at Asahigawasou Medical Welfare Academy, and has been an assistant professor 
of Health Informatics at Kawasaki University of M.W. since 2009.

Mihoko Okada received B.S degree in mathematics, M.S in computer science, and $\mathrm{Ph} . \mathrm{D}$ in medical science. She served as an assistant professor and then an associate professor of Statistics at Niigata University, and has been a professor of Health Informatics at Kawasaki University of M.W. since 1998.

Kazuko Yamamoto served as an assistant professor at the Department of Hygiene \& Public Health, Osaka Medical College, an associate professor at the Department of Environmental Health, Fukui Medical University (1983-1992), and a Professor at the Department of Medical Informatics, Shimane Medical University (1993-1999).

\section{Introduction}

Healthcare in Japan, as well as in many other countries, is facing many challenges, including delivery of efficient and high quality care, continuity of care, sustainable care, patient safety, cost containment, and so on. Information and communication technologies (ICT) play an increasing role for healthcare sectors, and advancement in Health and Medical Informatics has been and is being contributing greatly to better delivery of care. Introduction of Hospital Information Systems (HIS) started early in the 1970's in Japan. Patient accounting systems were introduced firstly, and now departmental systems and order entry systems are widely spread in hospitals all over the country. In the mid 1990's, computerized patient information processes were common, but patient records were not included among them, since healthcare institutions were required to maintain paper-based patient records and films for a prescribed period of time by a legal obligation. In view of the advances in ICT, the Japanese Ministry of Health and Welfare (MHW, currently the Ministry of Health, Labour and Welfare: MHLW) issued a notice on April 22, 1999 that allows patient records to be maintained on electronic media under a prescribed set of requirements.

Since then, Electronic Patient Record (EPR) systems have progressively introduced into hospitals. As more EPR systems are introduced into hospitals, the roles and responsibilities of the personnel in charge of patient record management are changing accordingly. Health information professionals should be conversant with not only patient records but also with the characteristics of electronic patient information, privacy and security, health information ethics, and so on. At the Department of Health Informatics, Kawasaki University of M.W., we have about 60 student enrollments each year, and about a half of them aim to work for hospitals as patient record administrators or as healthcare information technologists. For the students to prepare for EPR systems, we inaugurated the development of a computer based learning system called the "EPR Laboratory" in 2003. It is a self-learning system with a simulated environment for healthcare services delivery (Watanabe, Yamamoto, Okada, \& Takaue, 2005).

In medical and healthcare education, simulations have been introduced. In medical school, an education system for fostering student's problem solving ability during clinical practices was developed (Tsutsumi, Hori, \& Kuroda, 2003). Others have made use of real Electronic Patient Record systems for medical school student by controlling access for students (Hayashi, Nakagawa, Sugiyama, et al., 2005). In nursing departments, some education systems have been developed for teaching nursing diagnostics, nursing records, and so on (Takagi, Yoshioka, \& Matsunaga, et al., 2007). 
There is also a study that makes use of Web EPR systems, available as open source software (Kushniruk, Borycki, \& Armstrong, et al., 2009).

The EPR Laboratory has been developed to provide students with simulated environment for learning EPR systems in hospitals that are not easy to learn only from class room lectures. The functionalities of the EPR Laboratory are discussed in our previous paper (in Japanese) (Watanabe, Yamamoto, Okada, \& Takaue, 2005). The system is designed to supplement conventional lectures on health information systems given as part of our undergraduate curriculum. Using the Laboratory, the students may learn not only the operations of EPR systems but also the subjects connected with patient information handling, including privacy, security and health information ethics. It is basically meant for self-learning, but where appropriate, an instructor may give explanation. The EPR Laboratory is composed of an eLearing system and an EPR system. The learning materials are arranged in units in the eLearning system, and in each unit, the student learns the materials and the EPR operations through practice. Tests are given at each end of unit, and if a student failed a test, the system shows the student which questions were answered incorrectly and indicates which parts of the unit he/she should review. For this purpose, we introduced a structure to the learning materials on Health Information Ethics based on an information model (Okada, Hashimoto, \& Ohida, 2001; Okada, Yamamoto, \& Watanabe, 2007).

In the following, the overview of the system, the simulated environment to support learning of patient flow, information flow and hospital workflow, fundamental EPR operations, and structured learning materials for the test and review cycle are described.

\section{Background}

\subsection{Regulatory Actions in Japan}

The Japanese Ministry of Health and Welfare (MHW, currently the Ministry of Health, Labour and Welfare: MHLW) has been promoting a move away from paper-based to computer-based health care information management. Late in the 1990's, healthcare institutions were required to maintain paper-based patient records and films for a prescribed period of time by a legal obligation. The related laws and ministerial ordinances, including the Medical Practitioner Law, added up to ten. In view of the advances in ICT, the MHW issued a notice on April 22, 1999 that allows patient information to be maintained on electronic media under a prescribed set of requirements. Healthcare institutions are permitted to electronically maintain patient information written in documents which are obliged to be maintained legally under the following conditions (the following are tentative English translation from Japanese):

(1) Authentication and integrity shall be ensured.

- Appropriate countermeasures shall be taken to protect against intentional or accidental false entries, rewritings, deletions, and/or mixing.

- Accountability for electronically maintained patient information shall be ensured.

(2) Information shall be readable by sight.

- Patient information readable by sight shall be readily available upon request. 
- Patient information shall be readily printed upon request.

(3) Information shall be durable.

- Patient information shall be maintained during the period prescribed by the law in such a way that the contents are durable and reproducible during the entire period.

The action formed a part of deregulation efforts of MHW and it afforded healthcare providers an alternative method of maintaining patient information. It was expected that the notice would further promote the use of computers in the field of health care.

It has been well recognized that standards for healthcare information, including classification and coding, are imperative and standard development need be promoted further for electronically maintaining patient information. In April 2010, nearly ten years after the notification of electronically maintaining patient records, the MHLW issued a notice on the recommended standards of Health Informatics. Including are: HOT codes for pharmaceuticals, ICD10 compliant standard disease classifications, clinical information provision format for patient and patient referral (HL7 CDA compliant), IHE: PDI (Portable Data for Imaging) Integration Profile, and its application guideline, medical waveform format, DICOM, and laboratory information exchange protocol.

\subsection{Human Resources- Healthcare Information Technologist}

Hospital information systems and electronic patient record systems have become prevalent around the country, but there existed a number of problems as the systems in general are not flexible enough to produce various measures (indicators), hospitals are not able to propose their requirements for hospital information systems to vendors due to lack of expertise, and among other things, both hospitals and vendors lack human resources with specialties in health information systems and health information processing. There was an increasing recognition of the needs for health information professionals and for the cultivation of human resources in the healthcare sectors as well as in industry. In responding to social need, the Japan Association for Medical Informatics (JAMI) inaugurated the Healthcare Information Technologist (Healthcare IT) Certification in $2003^{1}$. A Healthcare IT, as a health and welfare professional, should be conversant with the special characteristics of healthcare and be capable of making use of and providing health information adequately and effectively, and employing information technology best suited to a given application. The first examination was given in August 2003. The number of examinees was 3,521, and 979 people passed the examination, and the overall pass rate was about $28 \%$. Since 2003 , certification examination is given annually. The certification is intended not only for healthcare professionals but also for people from industry including software engineers, managers, vendors, or others, involved in the fields of healthcare. Students are also among the expected examinees. As of June 2010, a total of 8,080 people are certified as Healthcare IT professionals. The certification "Senior Healthcare Information Technologist (Senior Healthcare IT)" started in 2007, and as of June 2010, 140 people are certified as Senior Healthcare IT professionals.

\footnotetext{
${ }^{1}$ http://www.jami.jp/hcit/HCIT_SITES/doc/pdf/090522HealthITOverview.pdf
} 


\section{Simulated Environment - Learning Healthcare Delivery at Hospital}

\subsection{Electronic Patient Record (EPR) Laboratory - Overview}

The Electronic Patient Record (EPR) Laboratory was developed on Caché ${ }^{\circledR}$ 5.0.11 (Caché is a registered trademark of InterSystems), using CSP (Caché Server Pages). Caché is installed as a Data Base management System in our computer rooms, and is connected to the Intranet. The system is employed in our course entitled "Health Data Management Practice" for the third year and fourth year students, using a computer room with one web server and 140 client PCs.

Figure 1 shows the overview of the EPR Laboratory. The system aims to support the students to learn not only operational aspects of EPRs but also fundamentals of healthcare service delivery and patient record management in hospitals. The system is comprised of the following: 1) an eLearning system and 2) an EPR system. The eLearning system is intended for students to acquire knowledge necessary to deal with patient information as patient information professionals. In the eLearning system, the learning materials are organized in units and the student walks through the Lab unit by unit. While the student is learning a unit, they are connected to the EPR system to learn fundamental operations through practices. The eLearning system also provides functions for instructors to prepare learning materials and tests, and to manage students' learning records.

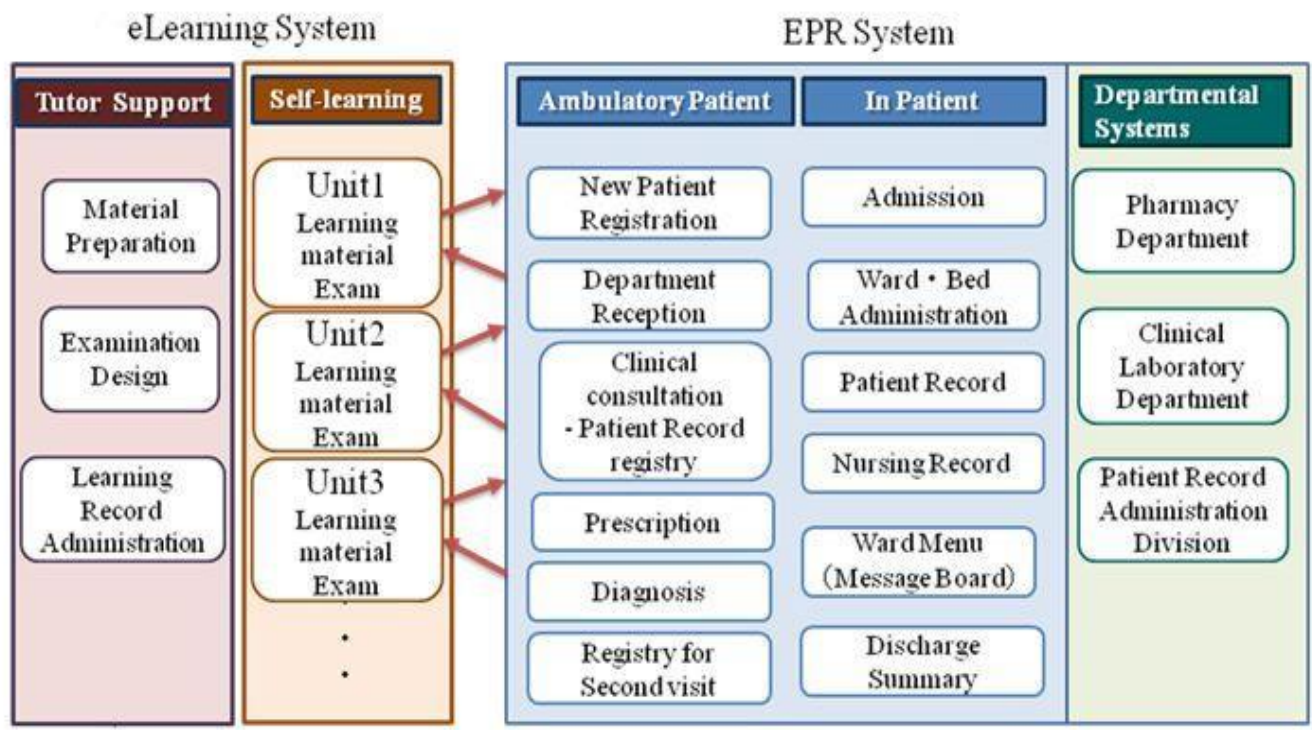

Figure 1. Overview of the EPR Laboratory

In the EPR system, there are four access modes: the doctor mode, the nurse mode, the clerical staff mode, and the patient record administrator mode. In the doctor mode, all functions of the EPR system are available. In the health record administrator mode, only limited operations are available, including patient profile data entry, ICD 10 code checking, creation and registration of template, hospital statistics processing, and so on. The EPR system is used only for educational purposes and is not a fully-fledged EPR system. 


\subsection{Walk through for an ambulatory Patient}

Figure 2 shows the initial screen of the beginning unit. English labels in the figures were prepared for this paper, as all messages are in Japanese in the actual system. In each unit, an objective(s), descriptions of given subjects, and tests are given. There is also a link to the corresponding part of the EPR system. The screen in Figure 2 starts with a fictitious story that a charming, curious elderly lady comes to visit the Hill-Top Hospital. The hospital is medium-sized, with 300 beds and about 300 ambulatory patients a day. The lady feels ill today and she wants to see a doctor. By clicking on "What you should know" in Figure 2, descriptions of hospital organization and functions are shown. Before moving the next unit, the student must go through a test by clicking on "Check your understanding" to check his/her knowledge. If they pass the test, then they can move to the next unit "General Information".

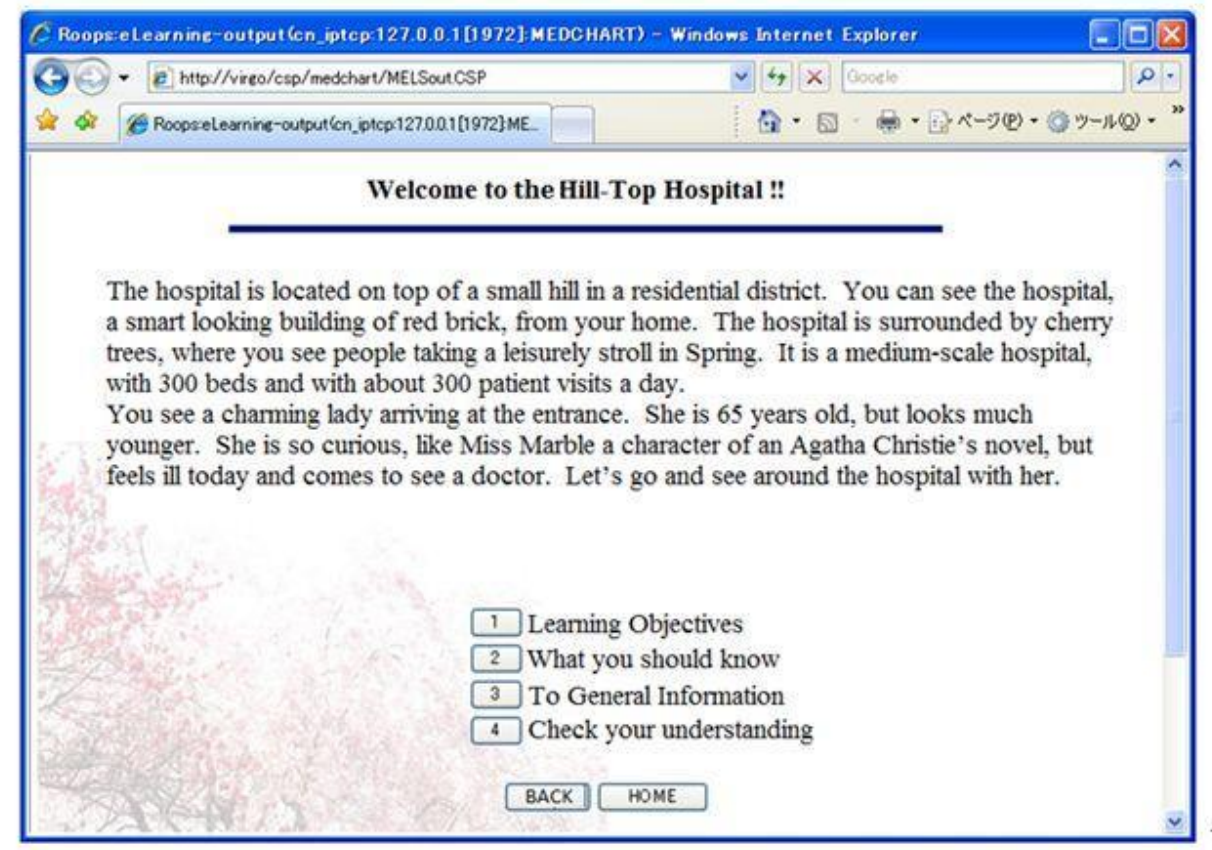

Figure 2. Initial screen of the eLearning system

Figure 3 shows the screen of "General Information". The student learns more details about the organization and function of a hospital, and the hospital accreditation system by Japan Council for Quality Health Care. By clicking on "Information Map and Organization of the Hospital," the student sees the map and description of each division. After passing the test, the student moves to the unit "Initial Visit Reception Desk".

Figure 4 shows the Initial Visit Reception Desk. Here the student learns about patient reception, patient information registry, ambulant patient and in-patient processes, and about the health insurance system. It is described that in Japan, patient registry is carried out at the accounting departmental system, and information is connected to an EPR system in general. At this point, the student learns about passwords, that is, they should change the password at regular intervals, what is a good password and what is not, that they should never write it on a strip of paper and post it around, and so on. Then the student logs into the EPR system under clerical staff mode, and enters the patient profile and health insurance information. Figure 5 is the screen of the patient profile data entry, 
where the student practices operations. After they finish data entry and came back to the eLearning unit, they must finish the test, and then move to Doctor's Office.

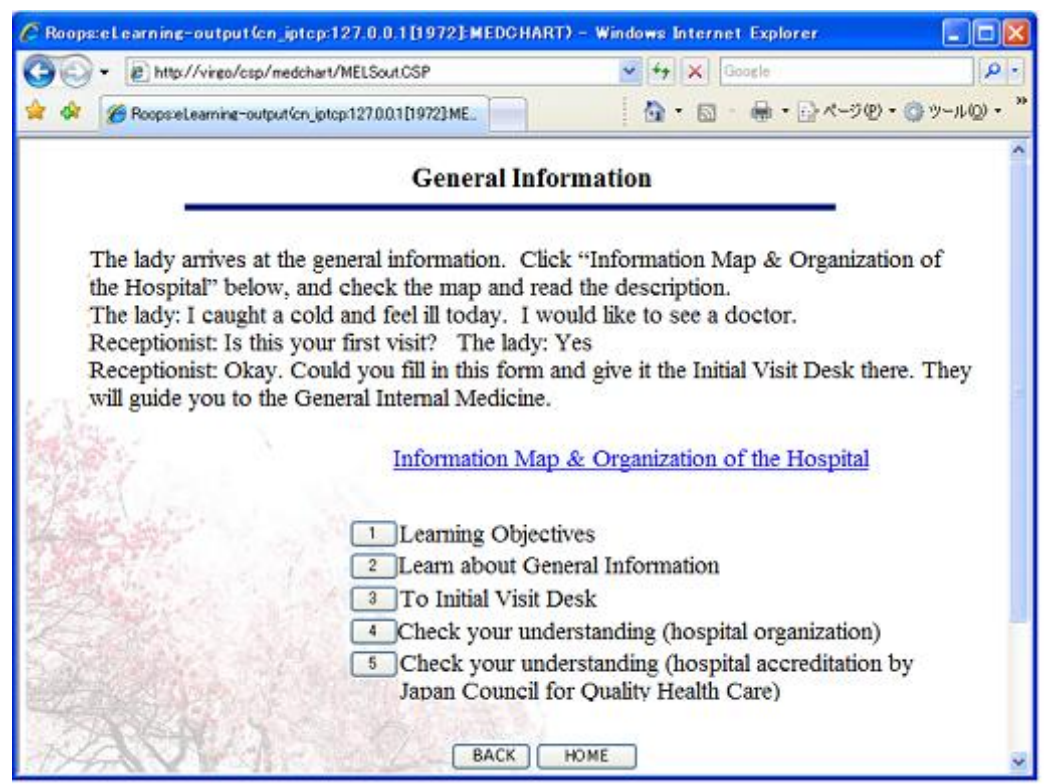

Figure 3. General information screen

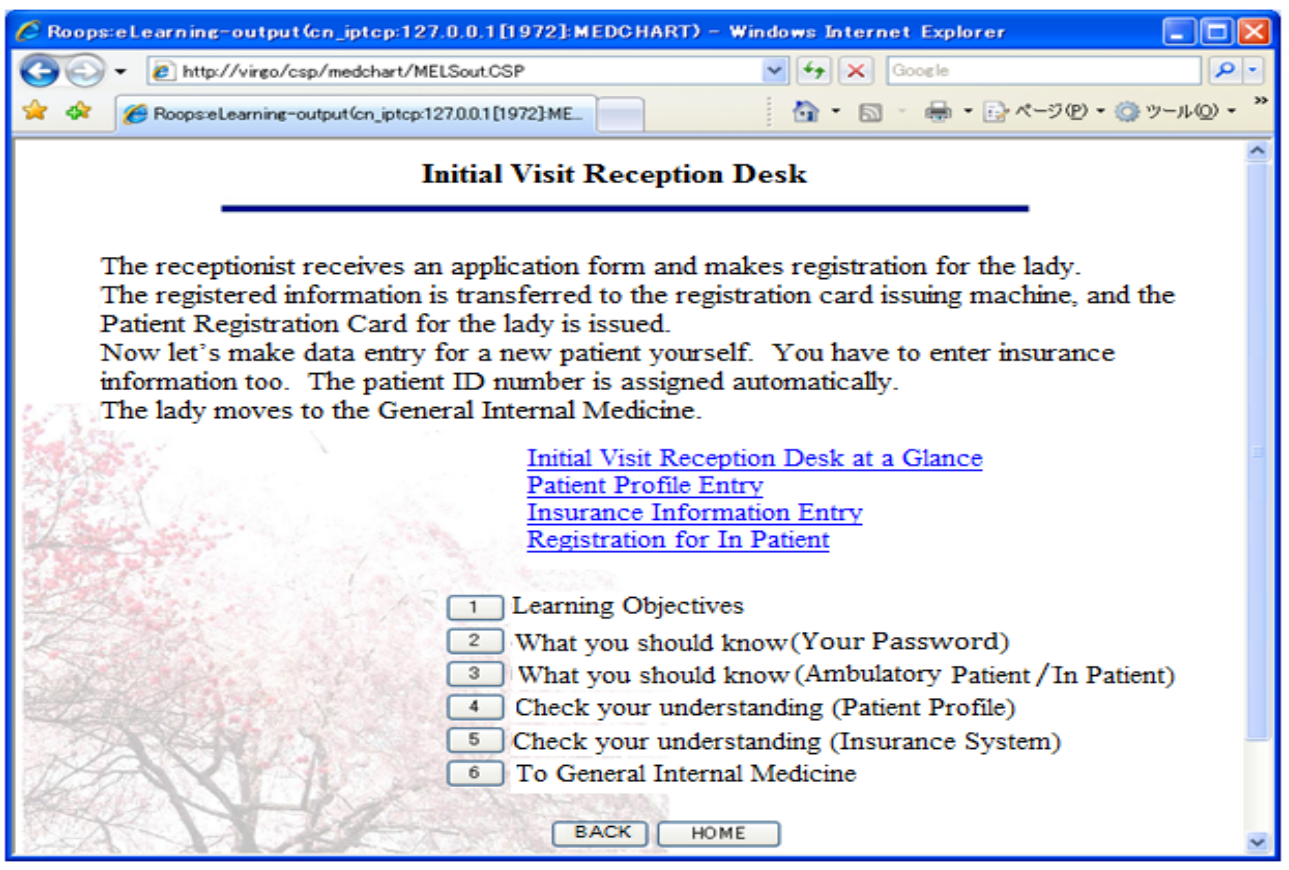

Figure 4. Initial visit reception desk 
The student logs off the EPR system and comes back to the eLearning system. Then they move to the Doctor's office (Figure 6). A dialogue between the doctor and the patient are shown on the screen. After the physical examination and tests, the doctor said that there is a chance of acute bronchitis. On this unit, the student learns hospital information system components, including the order entry system, pharmacy departmental and laboratory departmental systems, and the electronic patient record.

The student clicks on "Enter the EPR system as a doctor," and then logs on to the EPR system under the doctor mode. Then the screen of the EPR system appears, and they may view the existing data or enter data including progress notes, prescriptions and diagnosis. Figure 7 shows the screen for the diagnosis registry. Diagnoses may be entered using the search capability, and modifiers and sites (regions) may be added if necessary. Figure 8 is the screen for prescription data entry. Here the student learns about prescriptions by entering data including medicinal drugs, dosage, unit, and so on. They learn the notion of "do prescription" also.

Figure 9 is the screen of a patient record. Shown on the left hand side of the screen is the selection of forms, and the prescription history. The right had side is divided in to three frames, where shown at the top are patient demographics, in the lower left is the progress note and in the lower right are prescriptions.

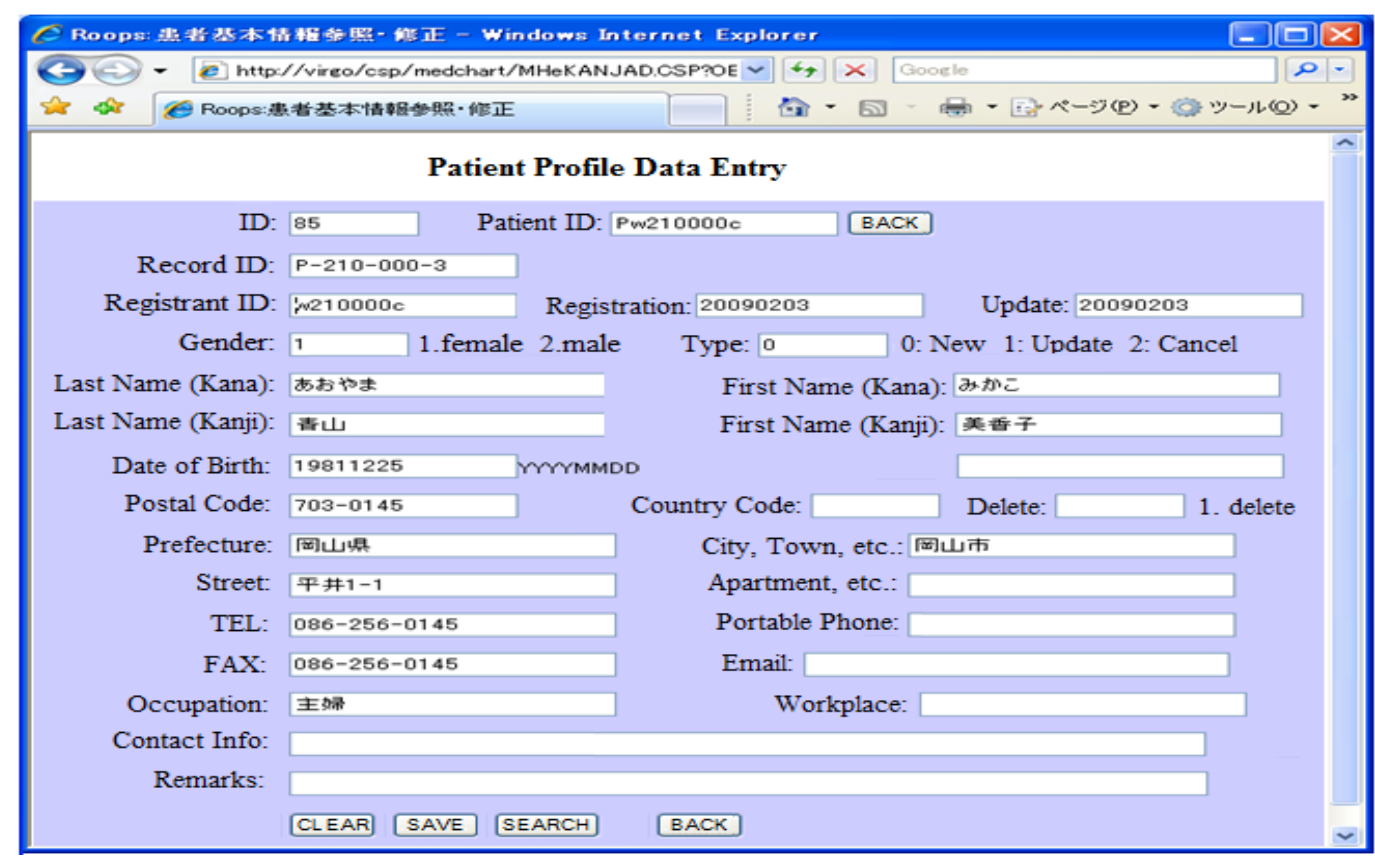

Figure 5. Patient profile data entry 


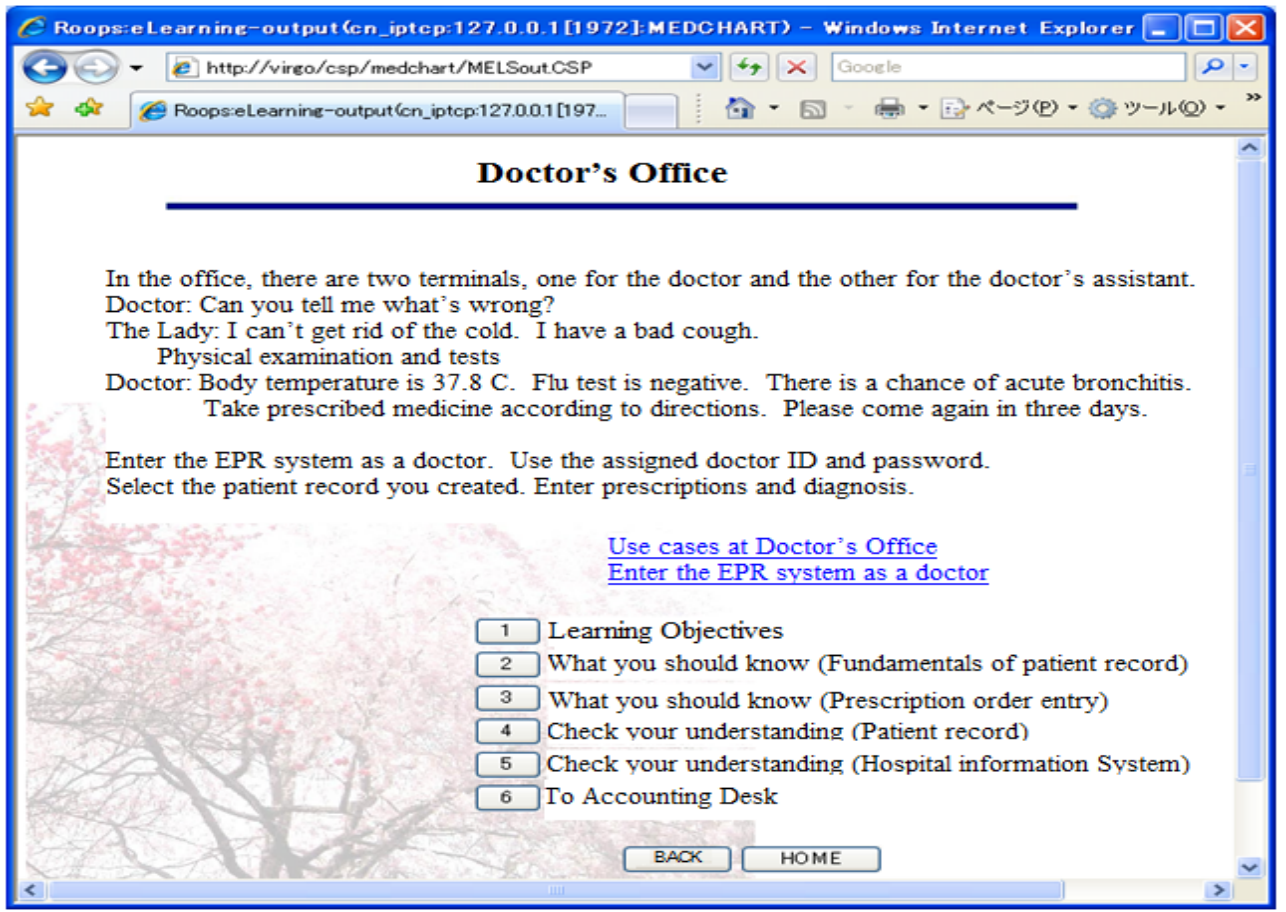

Figure 6. Doctor's office

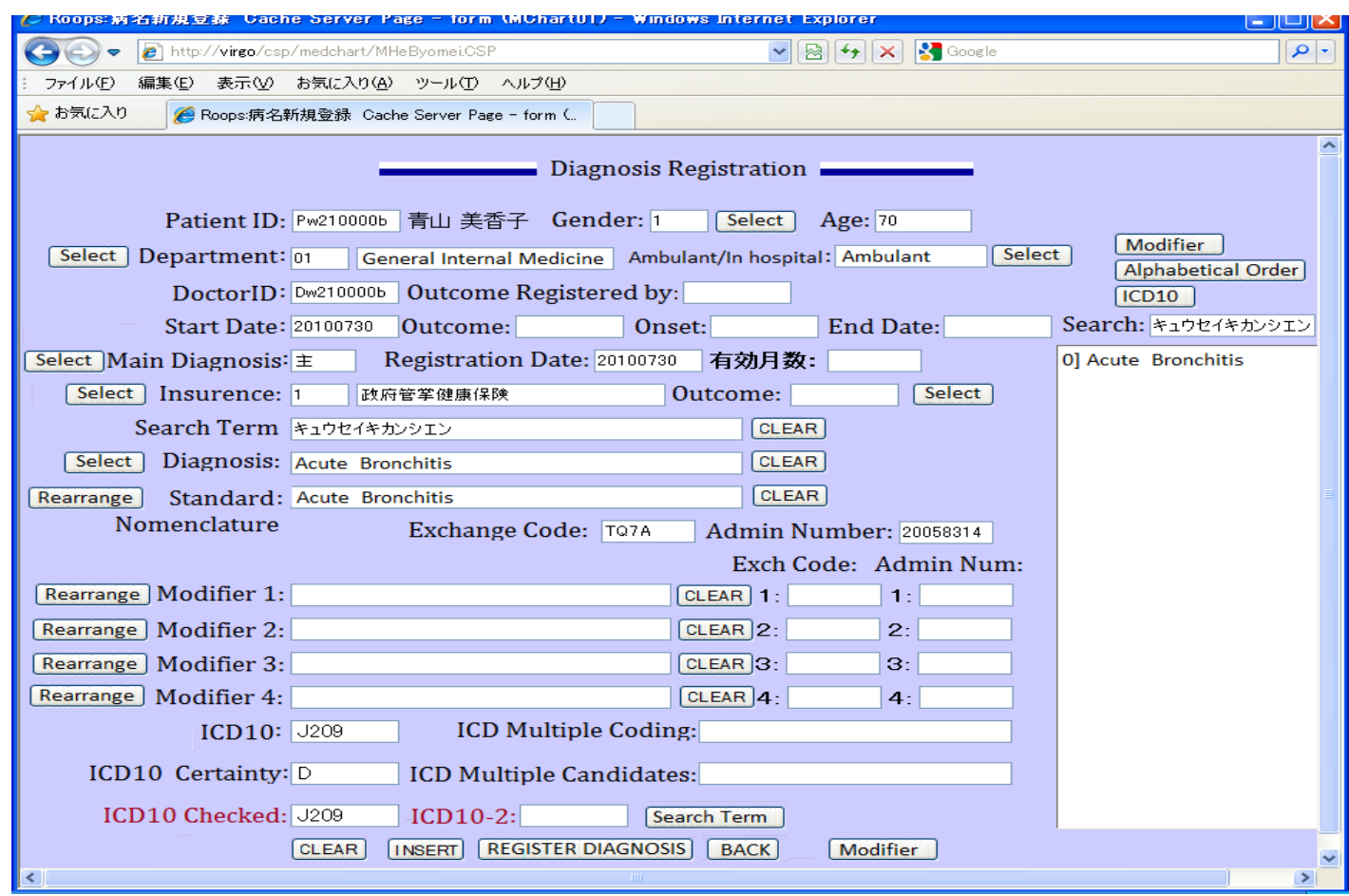

Figure 7. Registration of diagnosis 


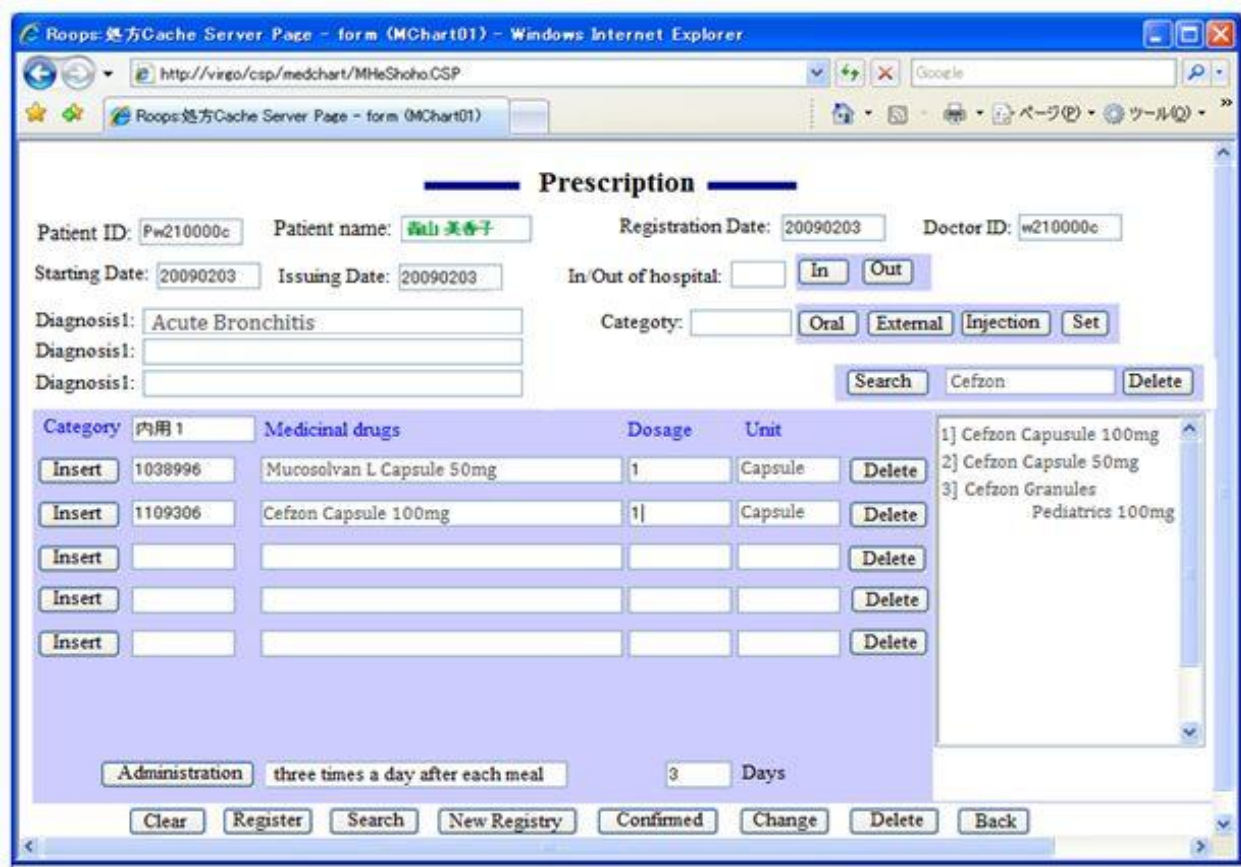

Figure 8. Prescription registry

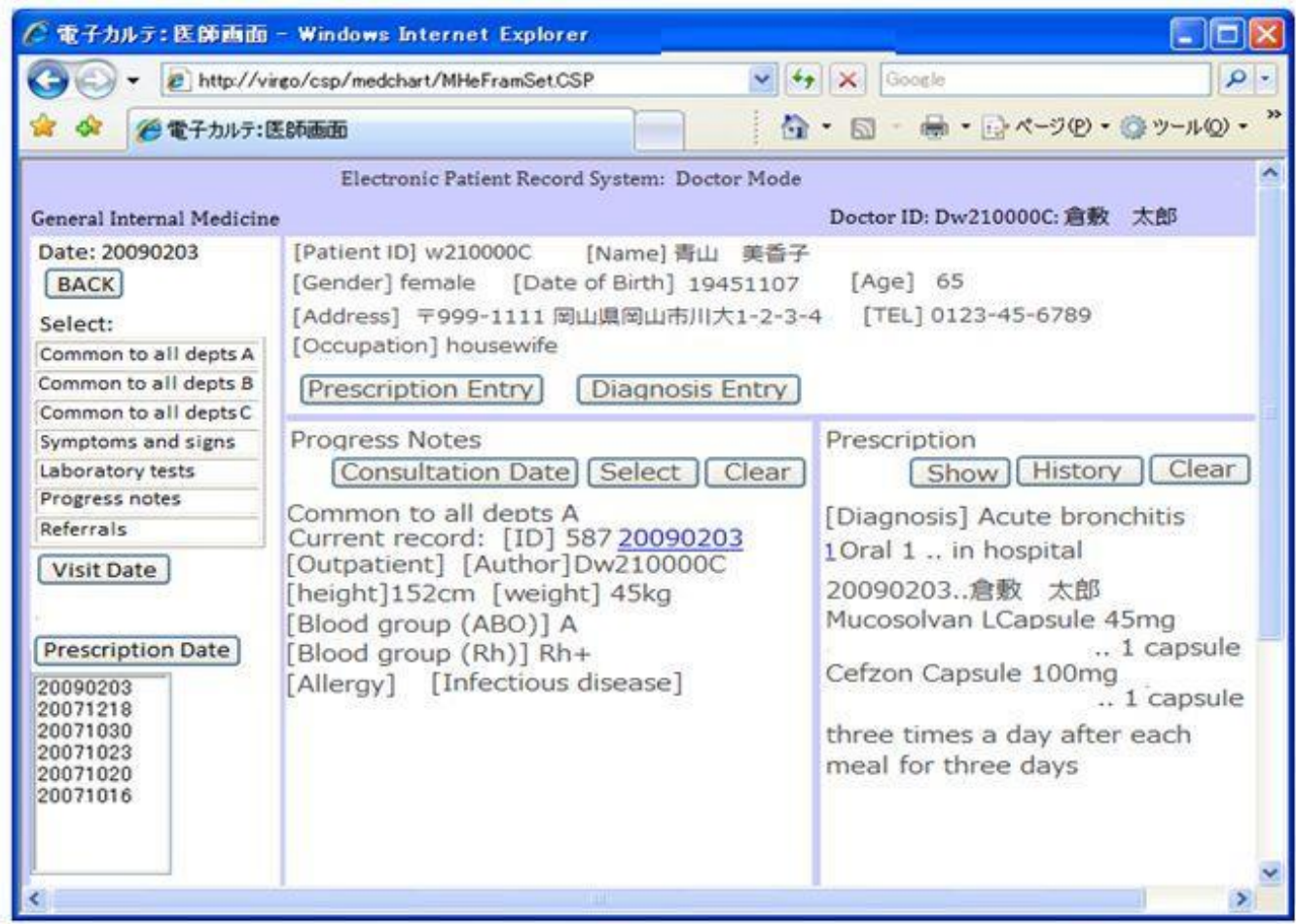

Figure 9. A screen showing a patient record 
In the Doctor Mode, the student also learns about templates. They create a template and enter the same information using the temple of his/her own. Through the practice, they learn data items differ from department and department, and different departments can use the same EPR system using templates.

After seeing the doctor, the patient then moves to pharmacy reception desk to receive prescribed drugs, and then moves to the Accounting Department. After ambulatory patients, the students learn the process for in patient. After the walk through, the students use the eLearning system and EPR system by themselves using sample cases. We use sample cases given in the guidelines for writing patient records published by Japan Medical Association.

\subsection{Patient Record Administration}

The EPR system has some departmental systems functionality (Figure 1). Since many of the students in our department aim to work for hospitals as health information professionals, we encourage them to obtain both certifications of Health Information Manager (HIM, the official naming was changed from Patient Record Manager to Health Information Manager), and Healthcare Information Technologist. We have special functionalities in the EPR system for patient record administration.

The students can also log on the EPR system under the health record administrator mode. Here they learn about ICD-10 coding on EPR systems, ICD-10 compliant Standard Master Files, hospital statistics processing (including indicators), patient referrals, and so on. In the EPR system, ICD-10 compliant Standard Master File (one of the recommended standards by MHLW) is installed, and when a diagnosis is registered, ICD10 code(s) are assigned automatically based on the Master File. The assigned code(s) are not necessarily correct, and the students playing the role of patient record administrator check whether the assigned ICD-10 code(s) are appropriate. If not appropriate, the students enter the proper ICD-10 code(s) in the field titled "ICD10 checked (Figure 7)." The corrected code must be confirmed by the doctor. For coding practices, advanced exercises may be taken for those who wish to be HIMs. We consider that the health information professionals need be acquired with health information standards, and through EPR Laboratory, they can learn the practicality of the Standard Master File and automatic coding, and correction of coding. They can also learn the standards for patient referral.

For statistical processing, general hospital statistics such as the number of patients by departments or disease classes, the average length of stay, bed occupancy rate, 5-year survival statistics by disease classes, etc. are computed. 


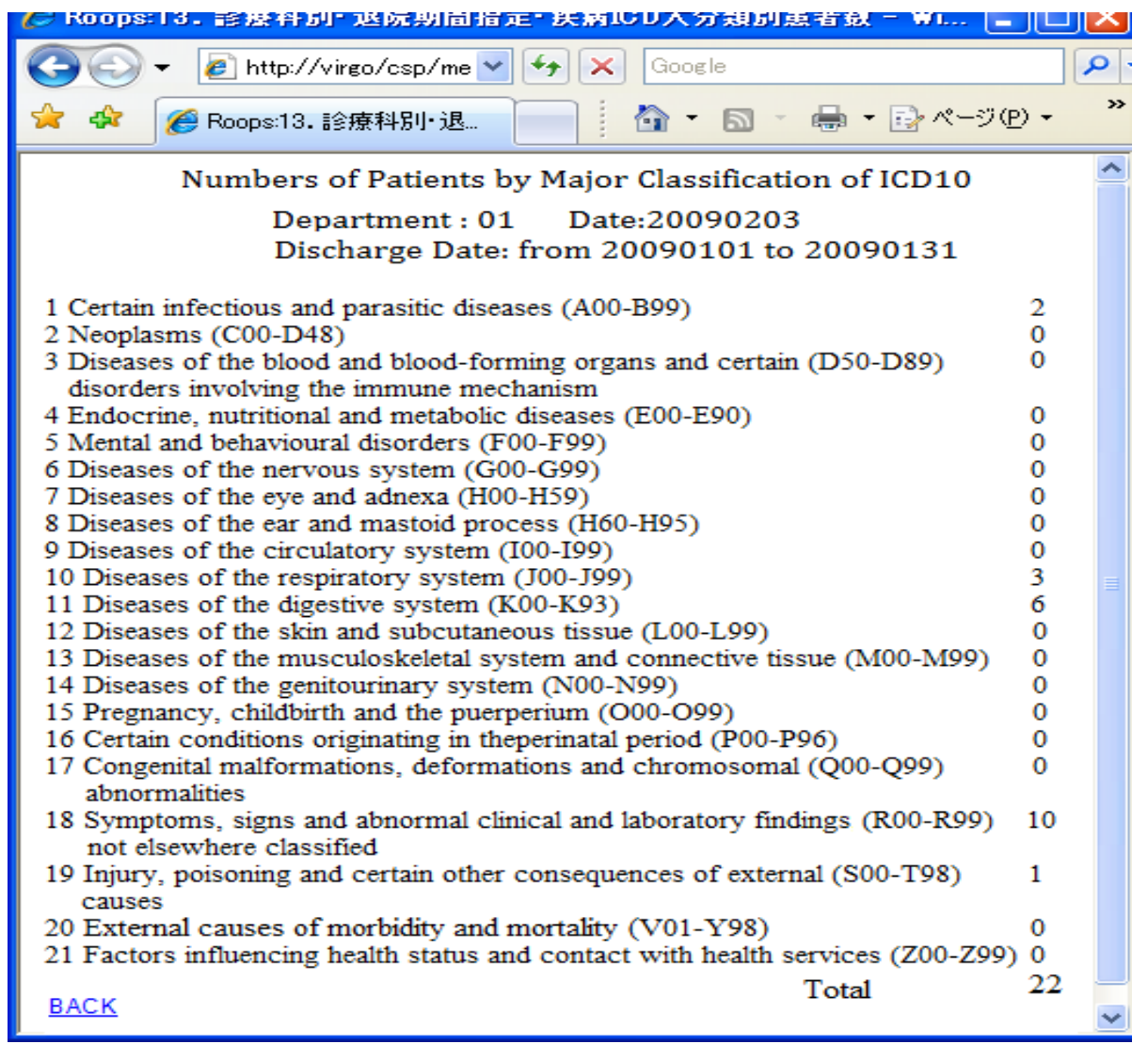

Figure 10. A sample screen of hospital statistics

\subsection{Test, Fail and Review Cycle}

The students must take a test(s) at the end of each unit. If they fail a test, they have to review the unit and take the test again (we call this the test, fail and review cycle). Evaluation criteria for the test may be set using the tutor's support functions of the eLearing system. A test is composed of questions, and questions may be given weight. If necessary, a question may be designated mandatory, that is, if the question is not answered correctly, then the test is in failure regardless of the total score.

When a student fails a test, he/she needs to know which questions they answered wrong. So when a student takes a test, the score and pass/fail are shown, and if he/she fails, the system shows the questions that were answered wrong. In each unit, the volume of materials may be heavy and it may not be necessary or may not be appropriate for the students to do it over. That is, it may be more desirable to show what materials should be reviewed. To support the test, fail and review cycle, we have introduced structure to the learning materials of the domain "Health Information Ethics" (Chapman, 1997; Harman, 2001). Included in the domain are the subjects strongly connected with the notion, including information security, privacy, ethics of clinical studies, EBM, etc. We consider that everyone who has access to information systems with patient data should learn the domain. We distinguish two levels of learning objectives: the first level for general users of health information systems, and the second level for those who are responsible for 
patient information handling, that is, "Health Information Professionals." We developed the teaching materials with education of the second level in mind. But selectively using the materials, the system may well be used by general users as well.

"Health Information Ethics" covers both healthcare services and medical (health) research. The scope is very wide, and some of the topics are very general and conceptual, and the others are narrow and concrete. Also many of the subjects or topics are related to one another in different ways. To represent the domain of "Health Information Ethics", we adopted the information modeling technique, and developed a conceptual model using UML. The model is used to give structure to learning materials including descriptive texts, published guidelines, exercises, and tests, and to give control mechanism to the test, fail, and review cycle in the EPR Laboratory.

The model consists of classes and relationships among them. There are three kinds of relationships: dependencies, generalizations, and associations. A "dependency" is used to show one thing using another. For example, there is a dependency from the class "EBM" to the package "Epidemiology," where a package is the basic grouping of things. We use a package when the contents are relevant but are broad in scope itself and taught elsewhere. An "association" is used to show structural relationships. An "aggregation" is a special kind of association and is used to model a "whole/part" relationship. Teaching materials including descriptive texts, exercises and tests are physical things and are considered objects, and each object belongs to a leaf class (a class with no children). Non-leaf classes are abstract classes (classes with no objects). For example, there are leaf classes "ResearcherEthicsGuideline," "ResearcherEthicsText," and "ResearcherEthicsTest." Belonging to the leaf class "ResearcherEthicsGuideline" are the actual guidelines including "World Medical Association Declaration of Helsinki Ethical Principles for Medical Research Involving Human Subjects," and the ethical guidelines published in Japan on medical or epidemiological research. The guidelines are referenced by the descriptive texts and the tests, and changes in the guidelines may affect the texts and the tests, but not the reverse, there are dependency relations from "ResearcherEthicsText" and "ResearcherEthicsTest" to "ResearcherEthicsGuideline." For leaf classes with tests as objects, the following attributes are defined. Here an object corresponds to a question:

$$
\begin{aligned}
& \text { class_name : the name of this class } \\
& \text { depend_on : the name of material(s) that this question depends on } \\
& \text { answer : an answer(s) to this question } \\
& \text { scoring_method : the method for scoring this question }
\end{aligned}
$$

The pass criteria for a test are specified by such rules as "the sum of all questions is greater than 80 and Question 1 is greater than 5 and Question 7 is greater than 5." When a student fails a test, the system shows which questions were answered wrongly and on which materials the questions depend on with a link to the materials. With this capability, a student is guided to the materials connected to the question(s).

There are three levels of questions: fundamental, advanced, and applied. A fundamental question is such that an answer can be found easily within a learning material. A question is advanced if it is related to multiple materials. A question is applied if an answer cannot be found directly in materials. If a student fails in answering fundamental or advanced questions, he/she is guided to read the relevant materials that are indicated by "depend_on" attribute. Shown in Figure 11 is the result of a sample test on information security. 


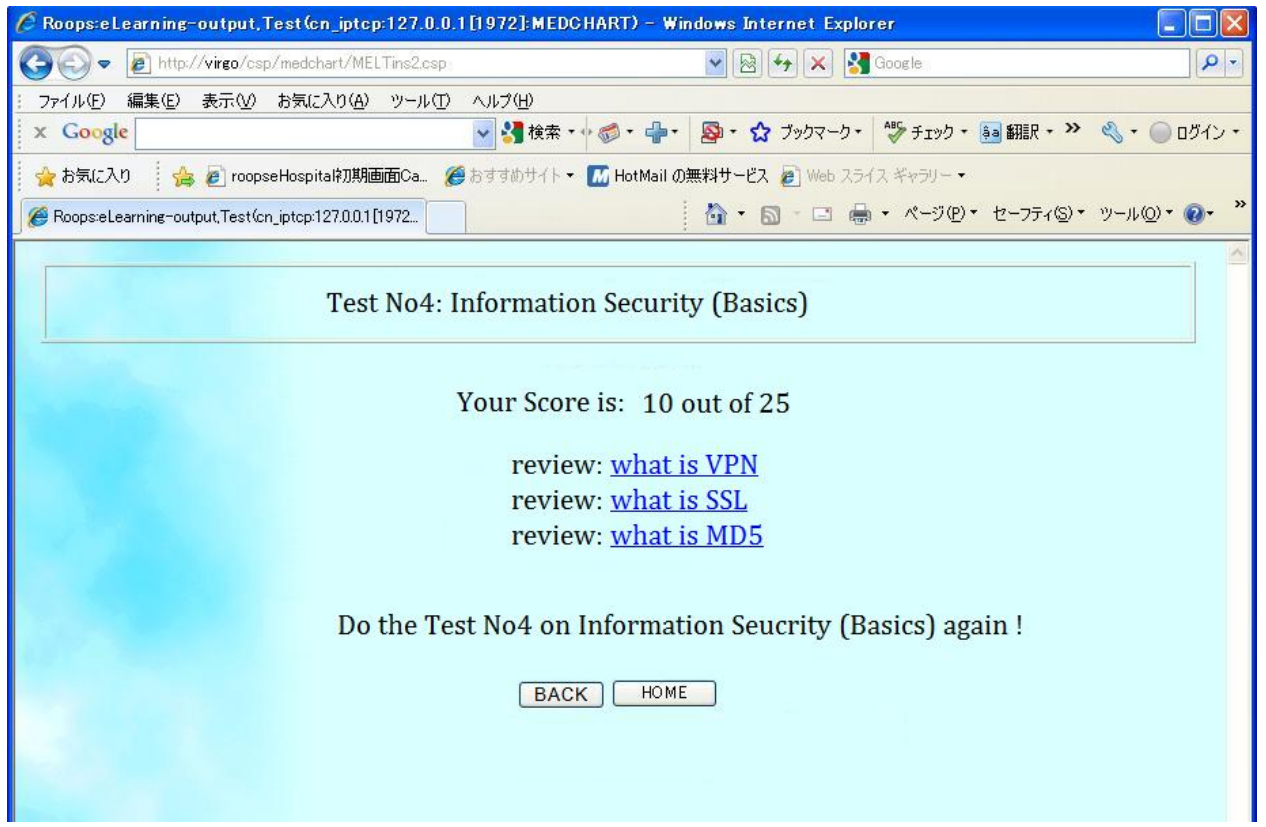

\section{Figure 11. The result of a test}

For fundamental and advanced questions, answers are selected from a list of choices, and the scores are computed automatically according to the rules specified by the attribute "scoring_method". Applied questions are given at near the end of the course. The questions are often taken from the real life problems which could be very complex. For this type of question, a student is asked to answer in writing in a textbox. The answer is not scored automatically but is sent to the instructor, and the score is given by the instructor. Two sample applied questions are shown below. The applied questions on personal data protection are adapted from Q\&A provided by MHLW on "Guideline on Healthcare and Welfare Entities Handling of Personal Information (2006, MHLW)".

A sample applied question 1:

Doctor "A" has left the Hill-Top Hospital. It is well known among the staff that his frank personality attracted patients, and there are some patients who are attached to him. Doctor "A" wants let the patients he was seeing know information about his new clinic and he asked the hospital that he would like to have a list of contact information of the patients. Do you think it is appropriate to provide the doctor with the information he requested? Discuss why you consider it appropriate or not appropriate.

A sample applied question 2:

Patient "A" has been hospitalized in the Hill-Top Hospital. The patient was referred to the Hill-Top from Doctor " $B$ " of another hospital. Doctor " $C$ " of the Hill-Top is in charge of the patient, and is communicating with Doctor " $B$ " for the best treatment of the patient. Now Doctor " $B$ " is requesting Doctor " $C$ " that he would like to obtain the patient record of "A" at the Hill-Top for his research purpose. Do you think it is appropriate to provide Doctor "B" with the information he requested? Discuss why you consider it appropriate or not appropriate. 


\section{Discussion and Conclusion}

There was an increasing recognition of the needs for health information professionals and for the cultivation of human resources in healthcare sectors as well as in industry. We consider that education is utmost importance to promote the adequate use of ICT in healthcare service delivery. The EPR Laboratory was developed for students who aim to become health information professionals to prepare for EPR systems. The system is selflearning and it provides simulated environment for healthcare services delivery that is not easy to learn only from attending class room lectures. It is comprised of the following components: 1) an eLearning system and 2) an EPR system. The eLearning system is intended for the students to acquire knowledge necessary to deal with patient information as patient information professionals including privacy, security and health information ethics. The learning materials are organized in units and the student walks through the Lab unit by unit. While the student is learning a unit, they are connected to the ERP system to learn fundamental operations through practice.

The learning materials are arranged in units, and in each unit, the student learns the materials and the EPR operations through practice. Tests are given at each end of unit, and when a student fails a test, the system shows which questions were answered incorrectly and indicates the related materials that they need to review, and then they try the test again. To support the test, fail and review cycle, we have introduced structure to learning materials of the domain "Health Information Ethics" based on a conceptual model using UML (Okada, Yamamoto, \& Watanabe, 2007). Based on the structure, when a student fails a test, the student may be guided to the materials that need be reviewed. In the domain of "Health Information Ethics," there are the subjects strongly connected with the notion, including information security, privacy, ethics of clinical studies, EBM, etc. We consider that everyone who has access to information systems with patient data should learn the domain. We developed the teaching materials for education of health information professionals, but by selectively using the materials, the system may well be used for education of healthcare professional in general.

It has been well recognized that standards for healthcare information, including classification and coding, are imperative and standards development needs be promoted further for electronically maintaining patient information. The importance of standards may not necessarily be obvious within one hospital, and to understand it, specialized knowledge of Health Informatics is necessary. In this regards, health information professionals are responsible for incorporating standards in their hospital. We have special functionalities in the EPR system for patient record administration. ICD-10 compliant Standard Master File (one of the recommended standards by MHLW) is installed in the system so that the students learn how the standards are used, why the standards are important, the limits of standards, and how health information professionals should be involved.

We consider that real ability for heath information handling may only be acquired through practice at healthcare settings, but at the same time, we consider that the students should acquire fundamental principles before they are involved in the real world decision making. The EPR Laboratory is developed for students aiming to become health information professionals but it may well be adopted for education of students planning to become healthcare professionals, or practicing healthcare professionals. We conclude that the system is useful for education about the current Electronic Patient Records that have characteristics different from paper-based records, not only from the operational and technical aspects, but also from health information ethics perspective. 


\section{References}

1. Chapman, A.R. (Ed). (1997). Health care and information ethics. Kansas City: Sheed \& Ward.

2. Harman, L.B. (2001). Ethical challenges in the management of health information. Maryland: Aspen.

3. Hayashi, R., Nakagawa, H., Sugiyama, E., et al. (2005). Procedure and Access Control for Allowing Medical Students to Access Electronic Patient Record Systems. Japan Journal of Medical Informatics, 25 (suppl.) (in Japanese).

4. Kushniruk, A.W., Borycki, E.M., Armstrong, B., Joe, R., \& Otto, T. (2009). Bringing electronic patient records into health professional education: towards an integrative framework. Studies in Health Technology and Informatics, 150, 883887.

5. Okada, M., Hashimoto, H., \& Ohida, T. (2001). Analysis and modeling to improve comparability of health statistics. Proceedings of MEDINFO 2001, Patel V. et al., eds. Amsterdam: IOS Press, pp.1374-1378.

6. Okada, M., Yamamoto, K., \& Watanabe, K. (2007). Conceptual model of health information ethics as a basis for computer-based instructions for electronic patient record systems. Proceedings of MEDINFO 2007, K. Kuhn et al. eds. Amsterdam: IOS Press, pp.1442-1446.

7. Takagi, H., Yoshioka, M., Matsunaga, A., et al. (2007). Development of electronic patient record training system for nursing practices - nursing database and care flow. Japan Journal of Medical Informatics, 27(suppl.), 330-333 (in Japanese).

8. Tsutsumi, H., Hori, A., Kuroda, N., et al. (2003). Usefulness of educational electronic patient record system for training of clinical practice. Medical Education, 34(6), 399-402 (in Japanese).

9. Watanabe, K., Yamamoto, K., Okada, M., \& Takaue, R. (2005). A computer aided instruction for electronic patient records in a hospital. Japan Journal of Medical Informatics, 25(4), 249-256. (published in March 2006, in Japanese). 